\title{
Modulo $2 \pi$ fringe orientation angle estimation by phase unwrapping with a regularized phase tracking algorithm
}

\author{
Juan Antonio Quiroga \\ Departamento de Óptica, Universidad Complutense de Madrid, Ciudad Universitarias S/N, 28040 Madrid, Spain \\ Manuel Servin and Francisco Cuevas \\ Centro de Investigaciones en Optica AC, Apartado Postal 1-948, 37150 León, Guanajuato, México
}

\begin{abstract}
Received November 30, 2001; revised manuscript received February 13, 2002; accepted February 25, 2002
The fringe orientation angle provides useful information for many fringe-pattern-processing techniques. From a single normalized fringe pattern (background suppressed and modulation normalized), the fringe orientation angle can be obtained by computing the irradiance gradient and performing a further arctangent computation. Because of the $180^{\circ}$ ambiguity of the fringe direction, the orientation angle computed from the gradient of a single fringe pattern can be determined only modulo $\pi$. Recently, several studies have shown that a reliable determination of the fringe orientation angle modulo $2 \pi$ is a key point for a robust demodulation of the phase from a single fringe pattern. We present an algorithm for the computation of the modulo $2 \pi$ fringe orientation angle by unwrapping the orientation angle obtained from the gradient computation with a regularized phase tracking method. Simulated as well as experimental results are presented. (c) 2002 Optical Society of America
\end{abstract}

OCIS codes: $100.2650,100.2960,120.3180,120.5050$.

\section{INTRODUCTION}

In many fringe-processing techniques a fringe pattern can be modeled in a first approximation as a phase-modulated sinusoidal irradiance distribution:

$$
I(\mathbf{r})=B(\mathbf{r})+M(\mathbf{r}) \cos \phi(\mathbf{r}),
$$

where $I$ is the irradiance, $B$ is the background, $M$ is the modulation, $\phi$ is the modulating phase, and $\mathbf{r}$ denotes the spatial dependence of these quantities. The fringe orientation angle can be defined as the angular direction of the gradient of the phase, that is,

$$
\beta_{2 \pi}(\mathbf{r})=\arctan \left[\frac{\partial \phi(\mathbf{r}) / \partial y}{\partial \phi(\mathbf{r}) / \partial x}\right] ;
$$

because of the arctangent function, this angle is computed modulo $2 \pi$, and it will be denoted by $\beta_{2 \pi}$. From the point of view of the fringe pattern, this orientation angle determines the perpendicular orientation to the fringe at each point. If the fringe pattern is normalized (the background is suppressed and the modulation is normalized), ${ }^{1-3}$ the fringe orientation angle can be computed from the normalized irradiance. The normalized version of the fringe pattern of Eq. (1) is

$$
I_{N}(\mathbf{r})=\cos \phi(\mathbf{r}),
$$

and from it the gradient is computed as

$$
\nabla I_{N}(\mathbf{r})=[\sin \phi(\mathbf{r})] \nabla \phi(\mathbf{r}) ;
$$

in consequence, for a normalized fringe pattern, the gradients of the phase and the irradiance are parallel but point in opposite directions every time $\sin \phi$ changes its sign. This change of sign occurs at the zero crossings of $\sin \phi$, that is, at locations with $\phi=k \pi, k$ being an integer. Also, the modulus of the irradiance gradient is modulated by $\sin \phi$. In other words, for normalized fringe patterns the gradient of the irradiance is parallel to the gradient of the phase, points toward the maxima of the fringe pattern, and at the maxima, the minima, and the stationary points of the phase is not well defined. When only one fringe pattern is available, Eq. (2) is not applicable and the fringe orientation angle must be computed from the gradient of the normalized irradiance [Eq. (4)]:

$$
\beta_{\pi}(\mathbf{r})=\arctan \left[\frac{\partial I_{N}(\mathbf{r}) / \partial y}{\partial I_{N}(\mathbf{r}) / \partial x}\right] ;
$$

because of the sign flips of the gradient's phase, this orientation angle is computed modulo $\pi$, and it will be denoted by $\beta_{\pi}$. In the general case of a fringe pattern with space-dependent background and modulation, it is still possible to compute the orientation angle by using Eq. (5), but the gradient of the irradiance will be, in general, not parallel to the gradient of the phase. ${ }^{4}$

Phase demodulation from single fringe-pattern images is important, for example, in the study of fast transient phenomena. In the case of fringe patterns with closed fringes, it is not possible to obtain good results with ordinary algorithms such as Fourier transform or spatial phase sampling methods. Recently, several studies that demonstrate the importance of the fringe orientation angle in reliable phase demodulation from a single fringe pattern have been published..$^{5-7}$ 
In the work of Servin et al., ${ }^{5}$ a fringe-following scanning strategy is employed together with a regularized phase tracking (RPT) technique to demodulate in a robust way speckle interferograms. In the RPT method the intensity of the fringe pattern is locally considered spatially monochromatic, so its irradiance may be modeled as a sinusoidal function phase modulated by a plane $p(\cdot)$. Thus, by minimizing an appropriate cost function that takes into account the differences between the measured irradiance and the proposed model, one can demodulate the phase $\phi(\mathbf{r})$ from the irradiance $I(\mathbf{r})$. In this case it is necessary to solve a nonlinear set of equations for every position $\mathbf{r}$ for the three parameters of the plane $p(\cdot)$; the phase $\phi$ and its local spatial frequencies $\omega_{x}=\partial \phi / \partial x$ and $\omega_{y}=\partial \phi / \partial y$. In a general scanning strategy, these parameters change from point to point, and thus the local phase and its spatial frequencies (or, equivalently, the modulo $2 \pi$ fringe orientation angle) are computed simultaneously. However, if a fringe-following scanning strategy is used, that is, if the fringe pattern is processed as much as possible along the isophasic lines, the value of the local phase $\phi(\mathbf{r})$ will change slowly from point to point, decoupling in some way its computation from that of the local spatial frequencies, making easier the demodulation.

In Ref. 6 Marroquin et al. adopt a different strategy but one with a similar philosophy. In this work the demodulation of a single fringe pattern is based on the construction of the analytic signal ${ }^{8}$ associated with the normalized fringe pattern $I_{N}(\mathbf{r})$, given by $A(\mathbf{r})=I_{N}(\mathbf{r})$ $+i \sin \phi(\mathbf{r})$. From it we demodulate the wrapped phase, computing it as $W(\phi)=\arctan [\operatorname{Im}(A) / \operatorname{Re}(A)]$, where $\operatorname{Im}($ ) and $\operatorname{Re}($ ) denote the imaginary and real parts, respectively, and $W$ denotes the wrapping operator ${ }^{9}$; that is, for any $\phi, W(\phi)=\phi+2 k \pi, k$ being an integer such that $-\pi<W(\phi)<\pi$. The analytic signal is computed by minimizing a global cost function that forces it to satisfy the following constraints: (1) The real part of $A(\mathbf{r})$ should be approximately proportional to the observed fringe pattern and should be locally monochromatic, and (2) the imaginary part of $A(\mathbf{r})$ should be close to the corresponding quadrature image of $I_{N}(\mathbf{r})$. As above, the local monochromatic character of the model implies that locally the phase field can be described by the value of the phase and its local spatial frequencies. As Marroquin et al. note, the simultaneous computation of the three parameters is computationally expensive and makes the minimization of the global cost function difficult and unreliable. The solution proposed in Ref. 6 is to decouple the computation of the local spatial frequencies from that of the local phase. In this approach the first step is the computation of the modulo $2 \pi$ fringe orientation angle. In their work Marroquin et al. propose a global regularizing scheme for this purpose, consisting of several global cost function minimization steps, which are difficult to implement.

Larkin et al. ${ }^{7}$ propose a totally different approach for phase demodulation from a single fringe pattern. In this work the so-called spiral phase quadrature $(\mathrm{SPQ})$ transform is presented. The main advantage of the SPQ transform is that it can be implemented as a standard linear filter and that it can be applied by using techniques such as a convolution or a Fourier transform. Given a normalized fringe pattern (3), the output of the SPQ transform applied to it is

$$
\mathrm{SPQ}\left[I_{N}(\mathbf{r})\right] \cong i[\sin \phi(\mathbf{r})] \exp \left(i \beta_{2 \pi}\right) .
$$

From relation (6) the result of applying the SPQ transform to a normalized fringe pattern is the corresponding quadrature term out of a phase factor given by the modulo $2 \pi$ fringe orientation angle. In consequence, to demodulate the phase by using an arctangent computation, it is necessary to cancel out this phase term by estimating it in some way.

The above discussion indicates that a reliable determination of the modulo $2 \pi$ fringe orientation is important for the direct demodulation of the phase from a single fringe pattern. In this work we present a new algorithm for the computation of the modulo $2 \pi$ fringe orientation angle by unwrapping the orientation angle obtained from the gradient computation. The unwrapping process is carried out by means of a modified RPT method, together with a fringe-following scanning strategy.

\section{THEORETICAL FOUNDATIONS}

\section{A. Phase Unwrapping Based on Regularized Phase Tracking}

In a general case a phase map $W(\phi)$ can be unwrapped, using the RPT procedure of Servin et al., ${ }^{10}$ by computing two signals in quadrature given by

$$
\begin{aligned}
& f_{C}(\mathbf{r})=\cos W(\phi)=\cos \phi ; \\
& f_{S}(\mathbf{r})=\sin W(\phi)=\sin \phi ;
\end{aligned}
$$

afterward, the phase of these signals is demodulated by minimizing at each point the cost function

$$
\begin{aligned}
& U_{\mathbf{r}}\left(\phi, \omega_{x}, \omega_{y}\right) \\
& =\sum_{(\xi, \eta) \in N \cap L}\left[\left|f_{C}(\xi, \eta)-\cos p(x, y, \xi, \eta)\right|^{2}\right. \\
& \quad+\left|f_{S}(\xi, \eta)-\sin p(x, y, \xi, \eta)\right|^{2} \\
& \left.\quad+\mu|\phi(\xi, \eta)-p(x, y, \xi, \eta)|^{2} m(\xi, \eta)\right]
\end{aligned}
$$

with

$$
\begin{aligned}
p(x, y, \xi, \eta)= & \phi(x, y)+\omega_{x}(x, y)(x-\xi) \\
& +\omega_{y}(x, y)(y-\eta)
\end{aligned}
$$

where $L$ is a two-dimensional lattice with valid data and $N$ is a neighborhood region around the coordinate $\mathbf{r}$ in the image where the phase is being determined. The field $m(\mathbf{r})$ is an indicator that equals 1 if the site has already been estimated and 0 otherwise, and, finally, $\mu$ is the socalled regularization parameter.

The first two terms of Eq. (8) correspond to the socalled fidelity term of the RPT method, and they reflect that the observations $f_{S}$ and $f_{C}$ are modeled as sinusoidal functions phase modulated by a plane $p(\cdot)$. This means that $f_{S}$ and $f_{C}$ are considered locally monochromatic; thus the parameters $\omega_{x}$ and $\omega_{y}$ can be interpreted as the local spatial frequencies of the phase $\phi$. The last part of Eq. (8) is the denominated regularization term, and it enforces smoothness and continuity of the estimated phase, 
making possible the computation of the continuous phase $\phi$. The smoothness of the result is controlled by the size of the neighborhood $N$ and the value of $\mu$. Typical values for $N$ and $\mu$ are $(5 \times 5)-(13 \times 13)$ and $1-10$, respectively.

To unwrap the phase map $W(\phi)$ and obtain the continuous phase $\phi$ by means of Eq. (9), we use the following method: Starting at a given location $\mathbf{r}_{0}$, are minimize $U_{\mathbf{r}}$ with respect to $\phi, \omega_{x}$, and $\omega_{y}$, obtaining $\phi\left(\mathbf{r}_{0}\right), \omega_{x}\left(\mathbf{r}_{0}\right)$, and $\omega_{y}\left(\mathbf{r}_{0}\right)$; in our case the minimization is performed by using a gradient descent algorithm. Once the minimization is performed, the location $\mathbf{r}_{0}$ is marked as processed, we set $m\left(\mathbf{r}_{0}\right)=1$ [initially, $m\left(\mathbf{r}_{0}\right)=0 \forall \mathbf{r}$ ], and we may proceed with the rest of the sites in $L$, repeating this operation from pixel to pixel following a given path.

\section{B. Phase Unwrapping of the Modulo $\pi$ Fringe Orientation Angle}

As explained in Section 1, from the normalized version of a fringe pattern, it is possible to obtain, by Eq. (5), the modulo $\pi$ fringe orientation angle $\beta_{\pi}$. The relation of $\beta_{\pi}$ to the modulo $2 \pi$ orientation angle is $\beta_{\pi}=\beta_{2 \pi}+k \pi, k$ being an integer such that $0 \leqslant \beta_{\pi} \leqslant \pi$; in consequence,

$$
W\left(2 \beta_{\pi}\right)=W\left(2 \beta_{2 \pi}+2 k \pi\right)=W\left(2 \beta_{2 \pi}\right) .
$$

Equation (10) states that the wrapped version of $2 \beta_{\pi}$ is equal to the wrapped version of $2 \beta_{2 \pi}$. Thus, by unwrapping $W\left(2 \beta_{\pi}\right)$, one can obtain $2 \beta_{2 \pi}$ and, simply by dividing this result by 2 , get the modulo $2 \pi$ fringe orientation.

The unwrapping of $W\left(2 \beta_{\pi}\right)$ has several problems. The first is that in the presence of closed fringes, $\beta_{2 \pi}$ is not a continuous function: If the center of a closed fringe is encircled, the fringe angle will present a continuous variation from 0 to $2 \pi \mathrm{rad}$, presenting a $2 \pi$ discontinuity at the starting point. These discontinuities start and finish at the maxima and the minima of the modulating phase and propagate across the fringe pattern, generating dislocations (with an amplitude of $2 \pi \mathrm{rad}$ ) in the fringe orientation angle. This means that in the presence of various extrema of the modulating phase, the fringe orientation angle will have several $2 \pi$ dislocations. In consequence, the $\beta_{2 \pi}$ angle can be considered a piecewise-continuous function, and any algorithm for its processing must take this into account. As we propose to unwrap $W\left(2 \beta_{\pi}\right)$, the natural $2 \pi$ dislocations are translated into $4 \pi$ ones, which must be preserved by the unwrapping algorithm. As an example, Fig. 1(a) shows a circular fringe pattern, and Fig. 1(b) shows the phase map $W\left(2 \beta_{\pi}\right)$; as indicated in Fig. 1(b), if the phase map is unwrapped along a circle containing the center of a closed fringe, the unwrapped phase will present a $4 \pi$ dislocation. Figure 1(c) shows a detail of the central region, indicating the location of the discontinuity sources and the $4 \pi$ dislocation.

The second problem is that because of the modulo $\pi$ computation of $\beta_{\pi}$ [Eq. (5)], two close discontinuity sources $^{9}$ of the same sign appear in $W\left(2 \beta_{\pi}\right)$, located at the centers of the closed fringes, at the start of the $4 \pi$ dislocations, as shown in Fig. 1(c). These discontinuity sources can be considered the sources of the dislocations. It is well-known that the discontinuity sources make the unwrapping path dependent. Also, they distort the cor-

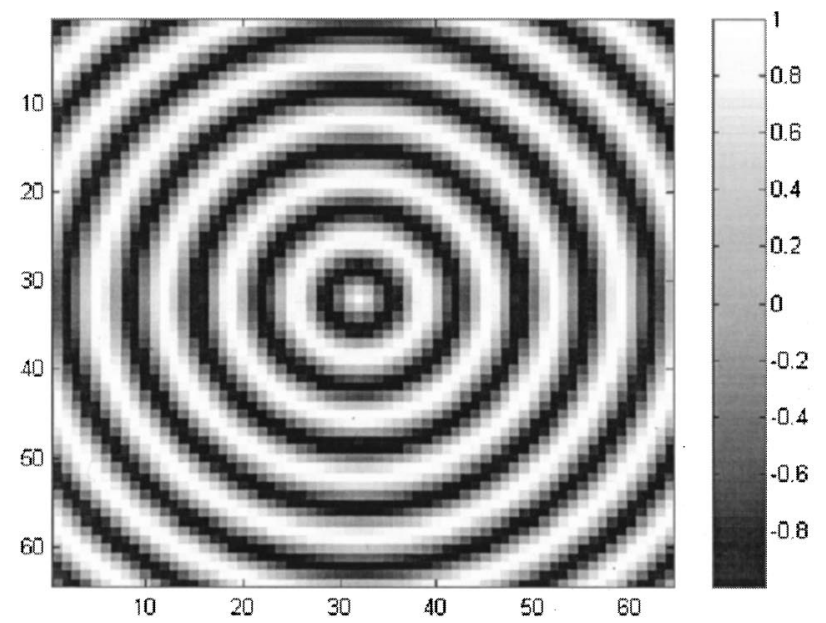

(a)

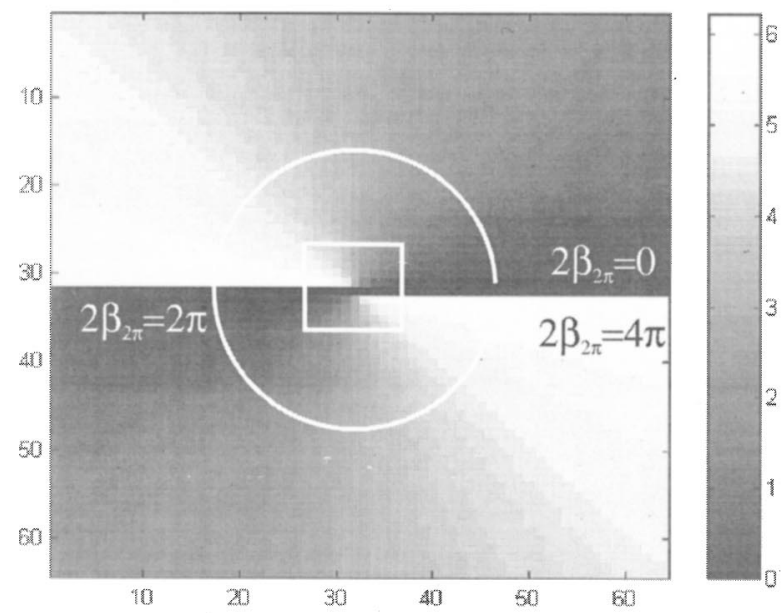

(b)

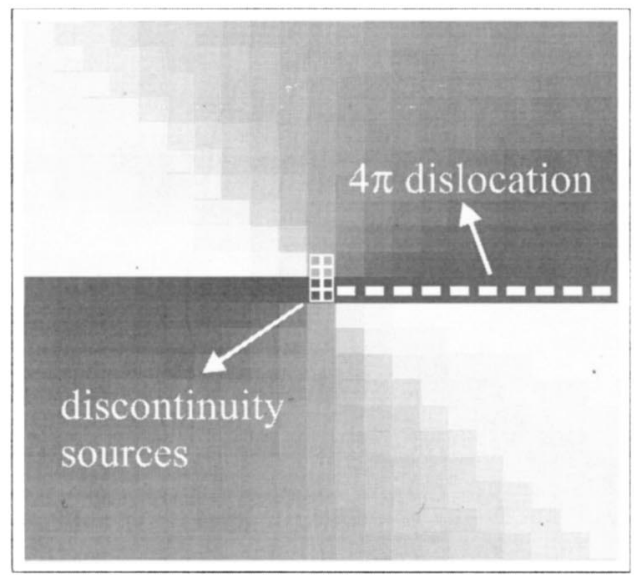

(c)

Fig. 1. (a) Noisy circular fringe pattern and (b) associated fringe orientation phase map $W\left(2 \beta_{\pi}\right)$, where black represents $0 \mathrm{rad}$ and white represents $2 \pi$ rad. As indicated in (b), if a circular path is followed, encircling the discontinuity sources, a total variation of $4 \pi \mathrm{rad}$ is found. (c) Amplification of the central white square of $W\left(2 \beta_{\pi}\right)$. The positions of the two positive discontinuity sources as well as the $4 \pi$ dislocation are marked. 


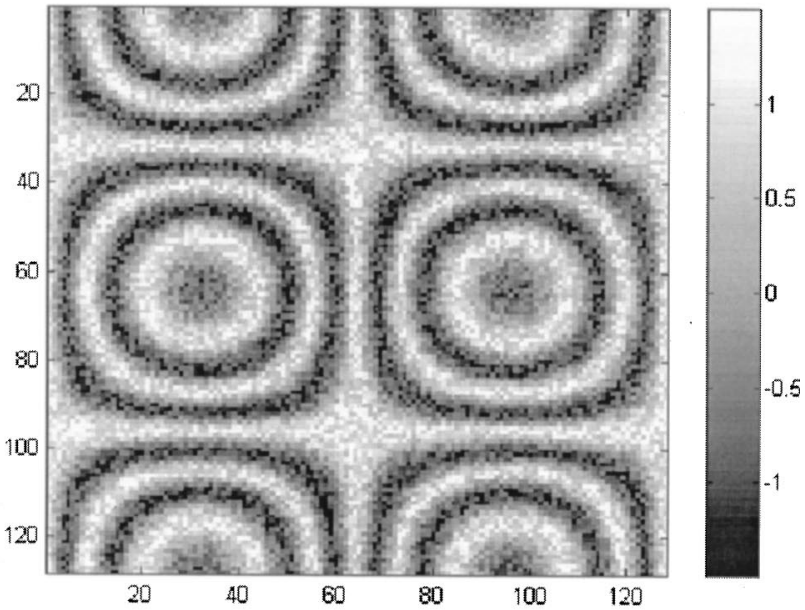

(a)

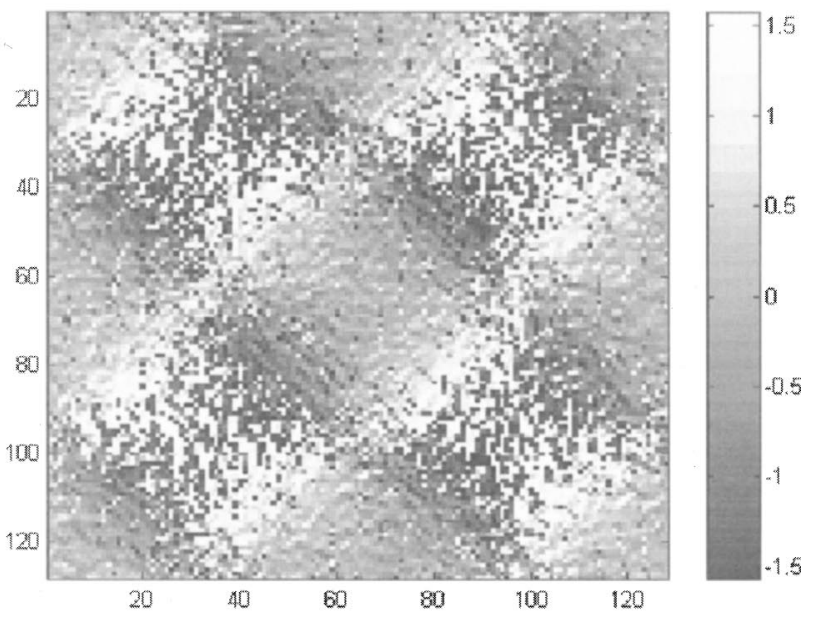

(b)

Fig. 2. (a) Computer-generated fringe pattern and (b) associated modulo $\pi$ fringe orientation phase map $\beta_{\pi}$, where black represents- $\pi / 2 \mathrm{rad}$ and white represents $\pi / 2 \mathrm{rad}$.

responding phase map in their neighborhood, making the phase unwrapping unreliable in their surroundings.

Finally, as $\beta_{\pi}$ is computed from the gradient of the irradiance, the noise present will be amplified by the derivative operation. Thus, in general, the phase map $W\left(2 \beta_{\pi}\right)$ will be noisy. This noise amplification is especially noticeable at those regions with low spatial frequencies, where both terms of the quotient necessary for the computation of $\beta_{\pi}$ are small. Then, at the centers of the closed fringes (maxima or minima of the modulating phase), superposed on the distortion effect of the discontinuity sources, big amounts of noise can be expected.

These three problems together make the phase map $W\left(2 \beta_{\pi}\right)$ logically inconsistent or, equivalently, its phase unwrapping path dependent; in consequence, a standard phase-unwrapping algorithm is precluded.

In this work we propose the use of a modified RPTbased phase-unwrapping algorithm. In this setting the phase unwrapping of $W\left(2 \beta_{\pi}\right)$ and the estimation of the modulo $2 \pi$ fringe orientation angle will consist in the demodulation of the quadrature signals given by

$$
f_{\beta C}=\cos W\left(2 \beta_{\pi}\right), \quad f_{\beta S}=\sin W\left(2 \beta_{\pi}\right),
$$

which, according to Eq. (10), corresponds to

$$
f_{\beta C}=\cos 2 \beta_{2 \pi}, \quad f_{\beta S}=\sin 2 \beta_{2 \pi} ;
$$

then $2 \beta_{2 \pi}$ is the modulating phase to be demodulated from the quadrature images given by Eq. (11). In this case the three parameters of Eq. (8) - $\phi, \omega_{x}$, and $\omega_{y}$-correspond to $2 \beta_{2 \pi}, 2 \partial \beta_{2 \pi} / \partial x$, and $2 \partial \beta_{2 \pi} / \partial y$, respectively.

With respect to the problem associated with the possible noise present in $f_{\beta S}$ and $f_{\beta C}$, it is well-known that $\mathrm{RPT}$ has a high signal-to-noise ratio in the estimated continuous phase. The reason for this is that the RPT system behaves as an extremely narrow-bandpass filter tracking a wideband fringe pattern signal. This property is especially remarkable in the case of the demodulation of two fringe patterns in quadrature, as it is in our case.

For the processing of the discontinuity sources of $W\left(2 \beta_{\pi}\right)$, it is possible to design an adequate scanning strategy. As mentioned above, the RPT demodulates in a sequential way the fringe pattern, starting at one seed pixel and then propagating from it until all of the region of interest has been processed. The way in which this se-

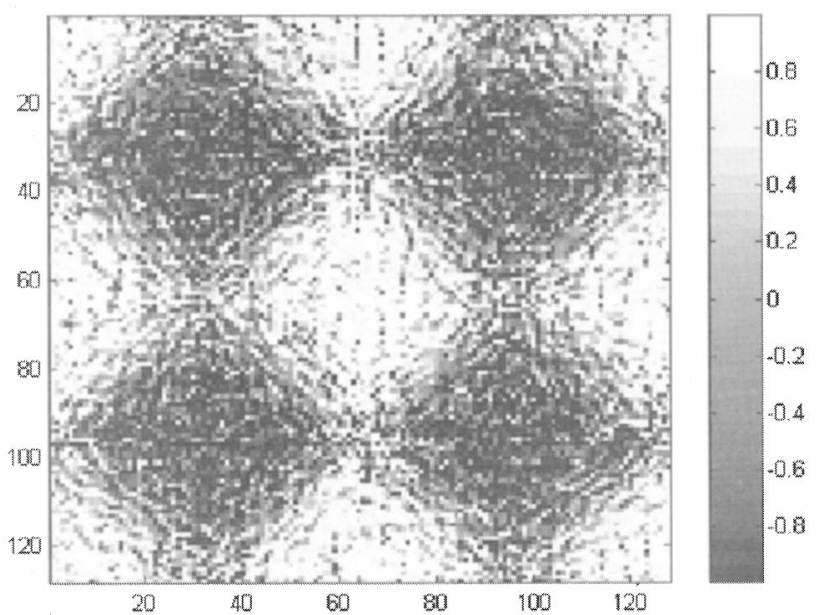

(a)

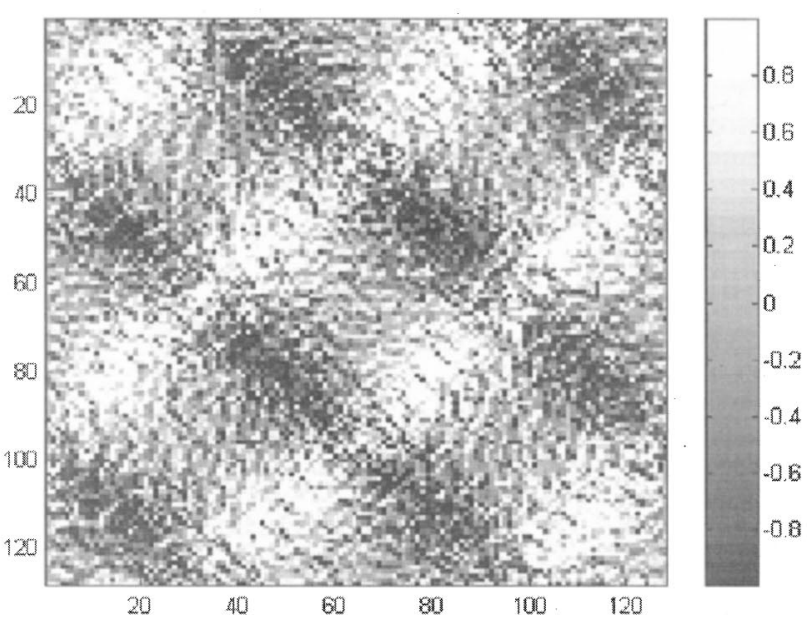

(b)

Fig. 3. (a) $f_{\beta C}$ and (b) $f_{\beta S}$ signals [Eqs. (11)] associated with the fringe orientation map $\beta_{\pi}$ shown in Fig. 2(b). 


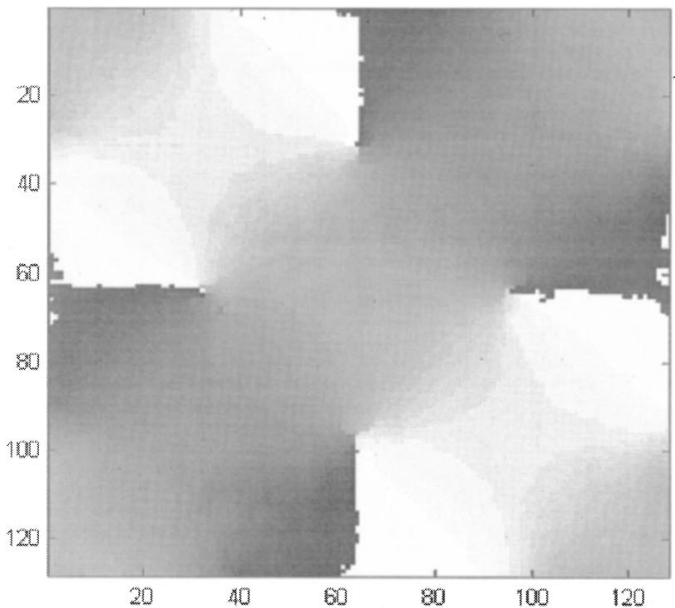

(a)

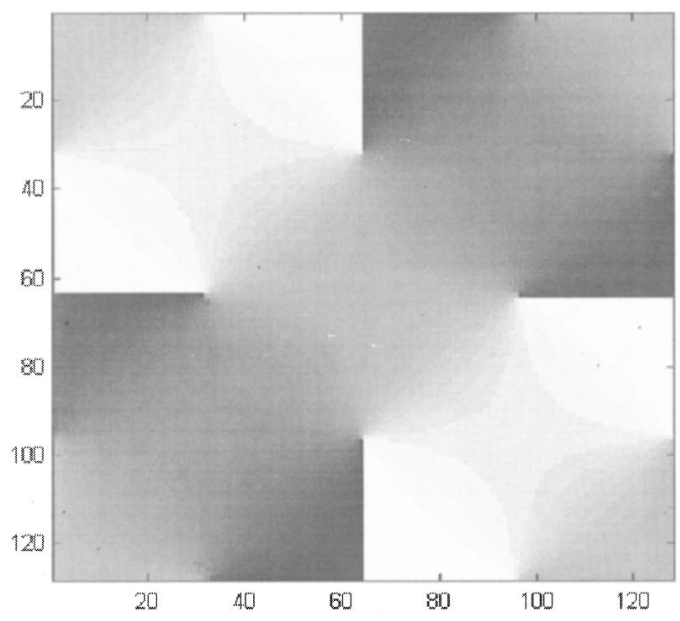

(c)
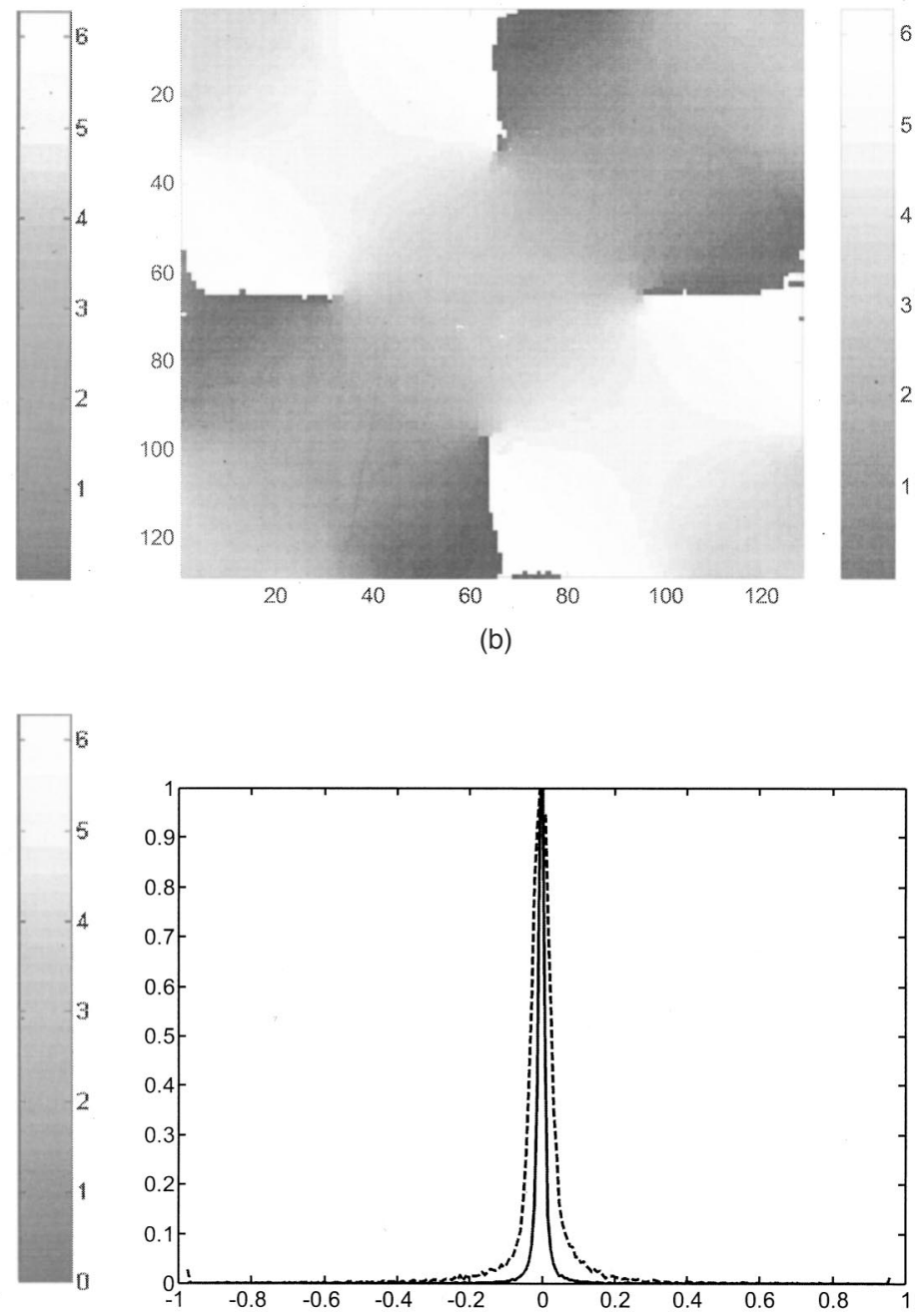

(d)

Fig. 4. (a) Modulo $2 \pi$ fringe orientation angle $\beta_{2 \pi}$ obtained with the modified RPT method for the fringe pattern of Fig. 2(a); in this case the fringe pattern was corrupted with additive noise normally distributed of standard deviation 1 . (b) Modulo $2 \pi$ fringe orientation angle $\beta_{2 \pi}$; in this case the fringe pattern was corrupted with phase noise normally distributed of standard deviation 1 rad. (c) Actual $\beta_{2 \pi}$ map corresponding to Fig. 2(a). (d) Histogram of the difference divided by $\pi$ between the estimated $\beta_{2 \pi}$ and the actual one for the two cases of additive noise (solid curve) and phase noise (dashed curve). In the phase maps of this figure, black represents 0 rad, and white represents $2 \pi \mathrm{rad}$.

quential scanning is performed has been demonstrated to be a key point in the demodulation process by means of RPT (actually, for any sequential image processing technique). The best choice that we have found is the use of a quality-map guided scanning strategy. Ströbel ${ }^{11}$ first proposed this in the context of phase unwrapping. The objective of the quality map is to guide RPT in such a way that it should first process locally the highest-quality areas, leaving low-quality regions to be processed last. In this way the possible errors that occur in the areas of low quality will be confined to these areas. In the case of $W\left(2 \beta_{\pi}\right)$, the discontinuity sources are located at the centers of the closed fringes; thus a natural choice for the quality map is the fringe pattern from which $W\left(2 \beta_{\pi}\right)$ is generated. In this case the quality map will guide the RPT unwrapping along the fringes encircling the discontinuity sources and always process these problematic points last; this procedure can be called isophasic scanning. For this reason, in the end, we adopted isophasic scanning for the processing of $W\left(2 \beta_{\pi}\right)$.
In spite of its advantages, the RPT-based phaseunwrapping method has a problem with respect to the discontinuous character of $\beta_{2 \pi}$ (the $2 \pi$ dislocations mentioned above). This is an important problem because we know that the orientation angle that we wish to recover can be discontinuous (if a closed fringe is present); on the other hand, the regularization term of the local cost function (8) imposes continuity. This problem can be solved by introducing a modification in the cost function (8). We know that if there are closed fringes, the phase $2 \beta_{2 \pi}$ is going to have $4 \pi$ jumps, so if we calculate the differences appearing in the regularization term of Eq. (8) modulo $4 \pi$, we are going to recover the continuous phase modulo $4 \pi$ associated with $2 \beta_{2 \pi}$. Then, for our modified functional, $4 \pi k$ and $4 \pi l$ represent the same value for all $k$ and $l, k$ and $l$ being integers. The basic assumption of this modification is that no noise is present with amplitude of $4 \pi$ rad or greater. In general, this is feasible, even for speckle interferograms, where phase-noise amplitudes of $1 \mathrm{rad}$ are expected. 
Finally, the modified local cost functional becomes

$$
\begin{aligned}
& U_{\mathbf{r}}\left(\phi, \omega_{x}, \omega_{y}\right) \\
& =\sum_{(\xi, \eta) N \in \neq L}\left[\left|f_{\beta C}(\xi, \eta)-\cos p(x, y, \xi, \eta)\right|^{2}\right. \\
& \quad+\left|f_{\beta S}(\xi, \eta)-\sin p(x, y, \xi, \eta)\right|^{2} \\
& \left.\quad+\mu\left|W_{4 \pi}[\phi(\xi, \eta)-p(x, y, \xi, \eta)]\right|^{2} m(\xi, \eta)\right],
\end{aligned}
$$

where $W_{4 \pi}$ denotes the modulo $4 \pi$ operation.

This procedure, a modified RPT-based phase unwrapping (including isophasic scanning) of the modulo $\pi$ fringe orientation angle, permits a robust method for the determination of the modulo $2 \pi$ orientation angle, as shown in Section 3.

\section{EXPERIMENTAL RESULTS}

The first example is a computer-generated fringe pattern. In this case the phase was computed as $\phi(i, j)$ $=15 \sin (2 \pi i / 128) \cos (2 \pi j / 128), i, j=1, \ldots, 128$, and from it a fringe pattern $I(\mathbf{r})=\cos \phi(\mathbf{r})+n(\mathbf{r})$ was generated,

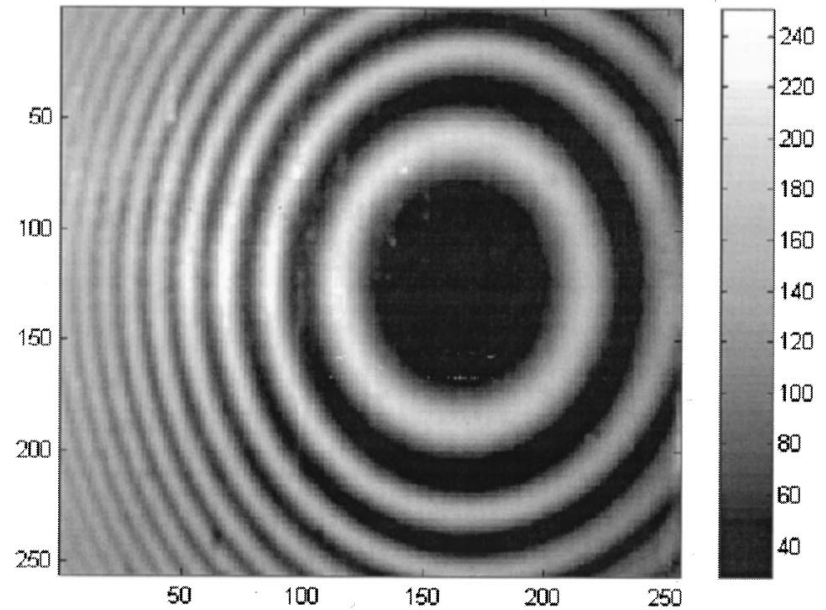

(a)

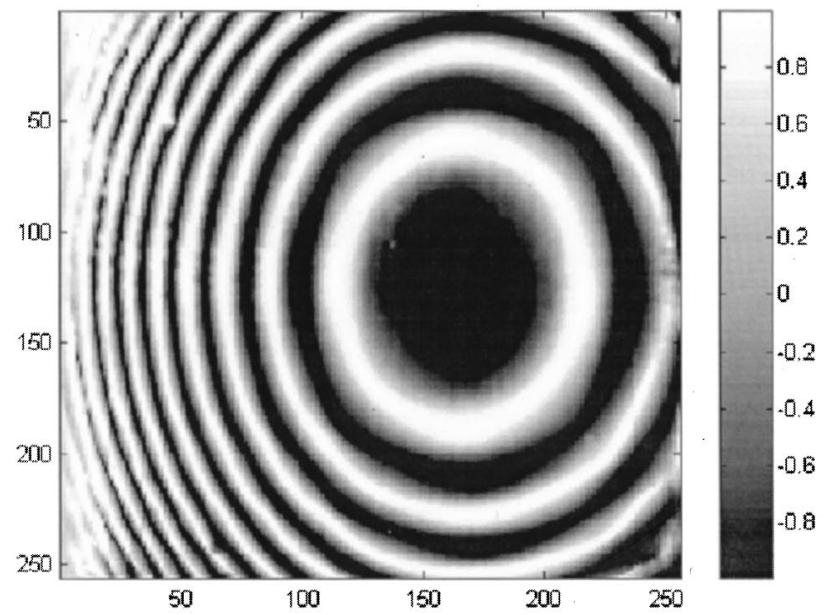

(b)

Fig. 5. (a) Shadow moiré fringe pattern. A clear variation from left to right of the background and the modulation is clearly visible. (b) Associated normalized fringe pattern.

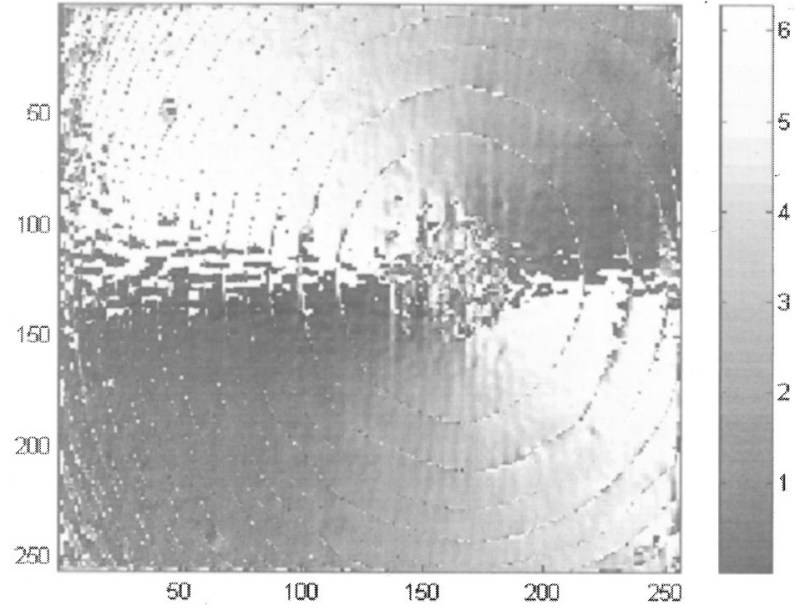

Fig. 6. (a) Fringe orientation phase map $W\left(2 \beta_{\pi}\right)$ computed from Fig. 5(b). Low-modulation regions associated with fringe maxima and minima are clearly visible; also, the center of the closed fringes (low-spatial-frequency region) shows a lowmodulation area. In this figure black represents 0 rad, and white represents $2 \pi \mathrm{rad}$.

$n(\mathbf{r})$ being a normally distributed random variable with standard deviation 1. For uncooled CCD cameras electronic additive noise with a normal distribution is the most important source of noise, and for this reason we have used it for the simulation. Figure 2(a) shows the noisy fringe pattern, and Fig. 2(b) shows the orientation angle modulo $\pi, \beta_{\pi}$, computed with Eq. (5). Figures 3(a) and $3(\mathrm{~b})$ show the quadrature signals $f_{\beta C}$ and $f_{\beta S}$, respectively, associated with $W\left(2 \beta_{\pi}\right)$, which have been computed with Eq. (11). Note the noise amplification in $\beta_{\pi}$ and, consequently, in $f_{\beta C}$ and $f_{\beta S}$. Figure 4(a) shows the modulo $2 \pi$ orientation angle $\beta_{2 \pi}$ computed by our method. To test the method with phase noise, we generated a fringe pattern $I(\mathbf{r})=\cos [\phi(\mathbf{r})+n(\mathbf{r})], n(\mathbf{r})$ being a normally distributed random variable with standard deviation $1 \mathrm{rad}$. Figure 4(b) shows the computed modulo $2 \pi$ orientation angle $\beta_{2 \pi}$. For comparison purposes Fig. 4(c) shows the actual fringe orientation angle computed with Eq. (2). In Fig. 4 one can clearly see the $2 \pi$ dislocations beginning at the centers of the closed fringes. Finally, Fig. 4(d) shows the histogram of the difference between the estimated $\beta_{2 \pi}$ and the actual one for the two cases of additive noise (solid curve) and phase noise (dashed curve). For the additive noise the mean value of the difference is $-0.002 \mathrm{rad}$, and the standard deviation is $0.04 \mathrm{rad}$. For the phase noise the mean value of the difference is $-0.004 \mathrm{rad}$, and the standard deviation is $0.12 \mathrm{rad}$.

The second example is an experimental shadow moiré fringe pattern obtained with a surface under deformation. Figures 5(a) and 5(b) show the fringe pattern and its normalized version, respectively. In Fig. 5(a) a clear background and modulation variation can be observed from left to right. For the normalization we used the algorithm described in Ref. 3. Figure 6 shows the $W\left(2 \beta_{\pi}\right)$ phase map computed from the normalized fringe pattern shown in Fig. 5(b). Low-modulation regions associated with fringe maxima and minima are clearly visible; also, the center of the closed fringes (low-spatial-frequency re- 
gion) shows a low-modulation area, source of the $2 \pi$ dislocation in $\beta_{2 \pi}$. Figures 7(a) and 7(b) show the quadrature signals $f_{\beta C}$ and $f_{\beta S}$, respectively, associated with the $W\left(2 \beta_{\pi}\right)$ phase map shown in Fig. 6. Figure 8(a) shows the modulo $2 \pi$ fringe orientation angle computed by our method. Again, the $2 \pi$ dislocation is visible, beginning at the center of the closed fringes. To validate this computation, we have demodulated the phase of the shadow moiré fringe pattern by using the SPQ transform [relation (6)] reviewed in Section 1, the result is shown in Fig. 8(b).

Finally, we have demodulated the phase from a single electronic speckle pattern interferometry (ESPI) fringe pattern by using the modulo $2 \pi$ fringe orientation angle obtained by our technique and the SPQ transform. Figure 9(a) shows the ESPI fringe pattern. Figure 9(b) shows the modulo $2 \pi$ fringe orientation angle $\beta_{2 \pi}$ computed by our method. Figure 9(c) shows the demodulated phase with use of the SPQ transform [relation (6)]. For comparison purposes Fig. 9(d) shows the cosine of the phase map shown in Fig. 9(c) as can be seen, the result is close to the fringe pattern shown in Fig. 9(a).

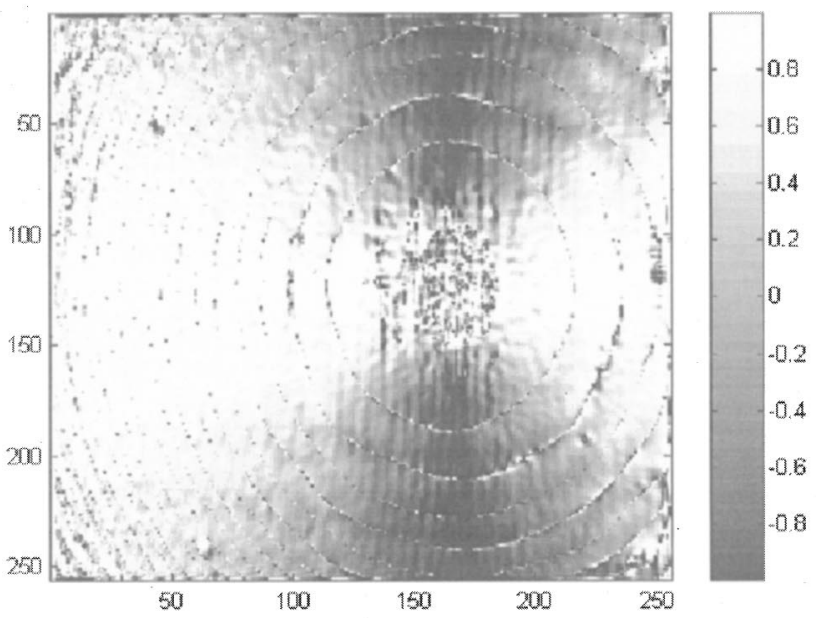

(a)

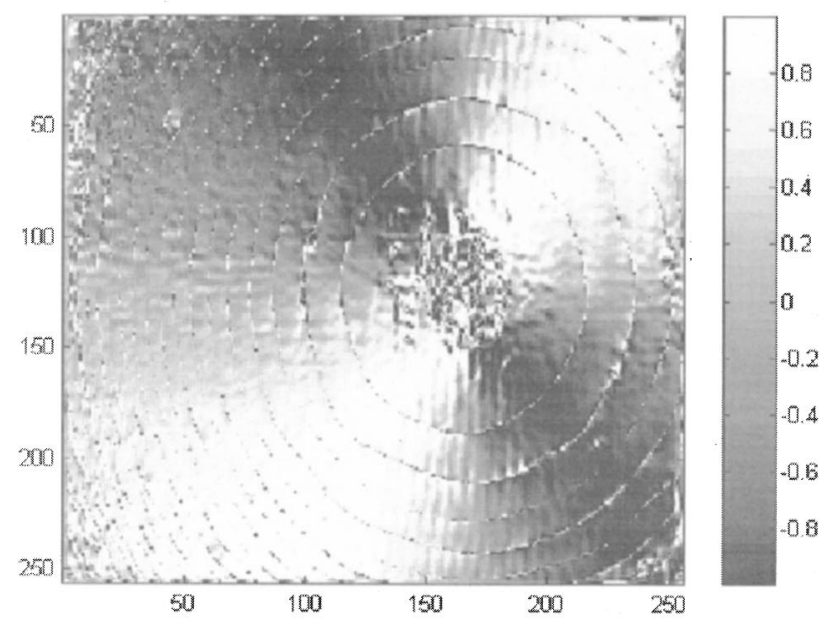

(b)

Fig. 7. (a) $f_{\beta C}$ and (b) $f_{\beta S}$ signals [Eqs. (11)] associated with the fringe orientation phase map $W\left(2 \beta_{\pi}\right)$ shown in Fig. 6 .

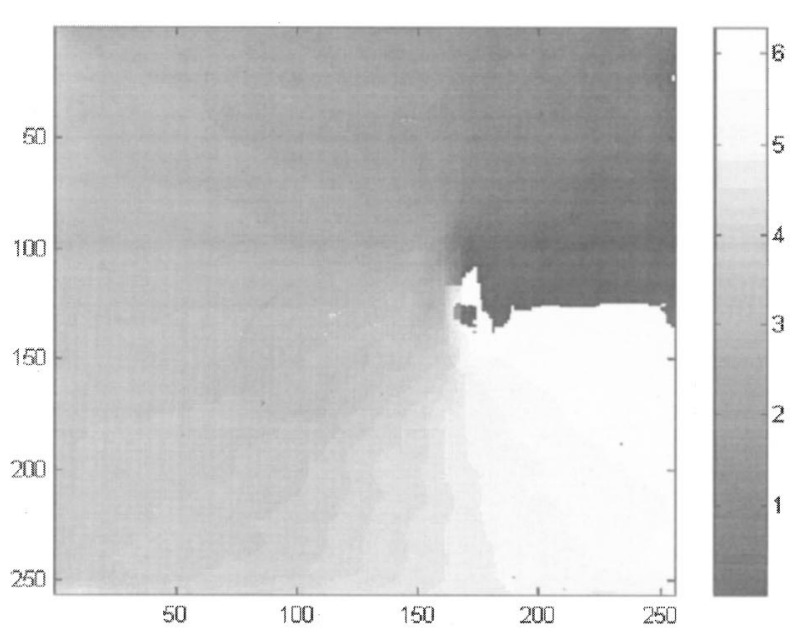

(a)

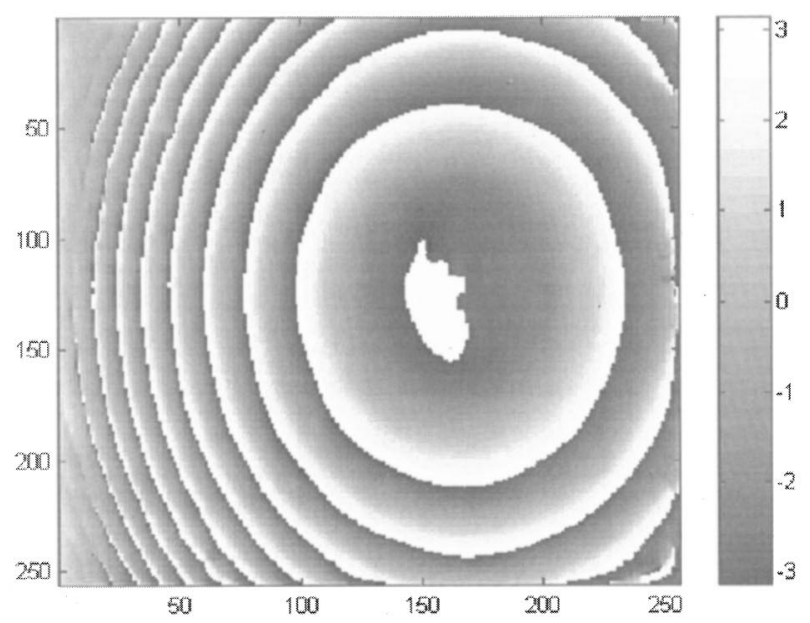

(b)

Fig. 8. (a) Modulo $2 \pi$ fringe orientation angle $\beta_{2 \pi}$ obtained by the modified RPT method for the fringe pattern of Fig. 5 , (b) demodulated phase map obtained by using the SPQ transform [relation (6)] applied to the fringe pattern of Fig. 5 with the orientation angle in (a).

\section{CONCLUSIONS}

We have presented a new method for the computation of the modulo $2 \pi$ fringe orientation angle of a single fringe pattern by unwrapping the orientation angle modulo $\pi$ obtained from the gradient computation. The unwrapping process is carried out by means of a modified RPT method, together with a fringe-following scanning strategy. The modified RPT phase-unwrapping process takes into account the $2 \pi I$ discontinuities that appear in the modulo $2 \pi$ fringe orientation angle when closed fringes are present. In the presence of closed fringes, the phase dislocations of the orientation angle modulo $2 \pi$ preclude the use of conventional phase-unwrapping algorithms such as the methods described in Refs. 10 and 11. This technique, together with the SPQ transform of Larkin et $a l .{ }^{7}$ permits a robust demodulation of the phase from a single fringe image with closed fringes. Simulated as well as experimental results have been presented with good results. 


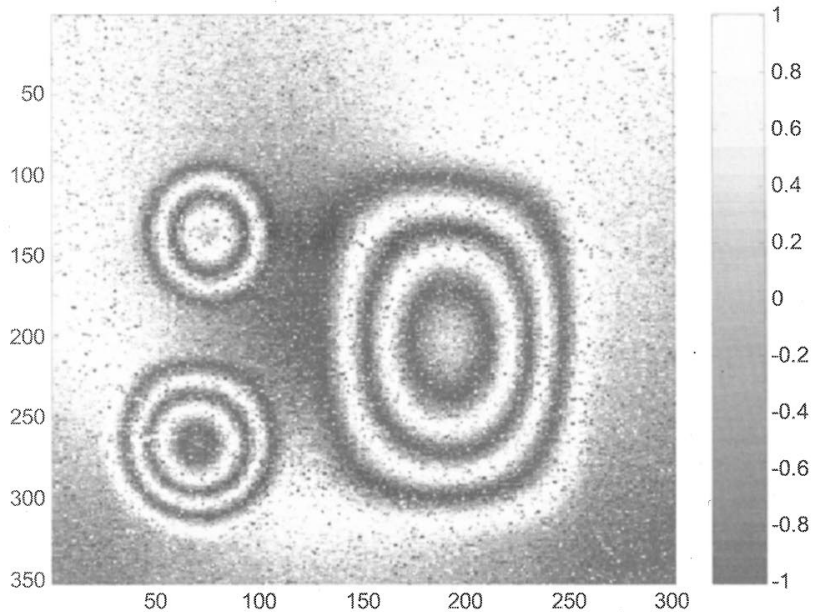

(a)

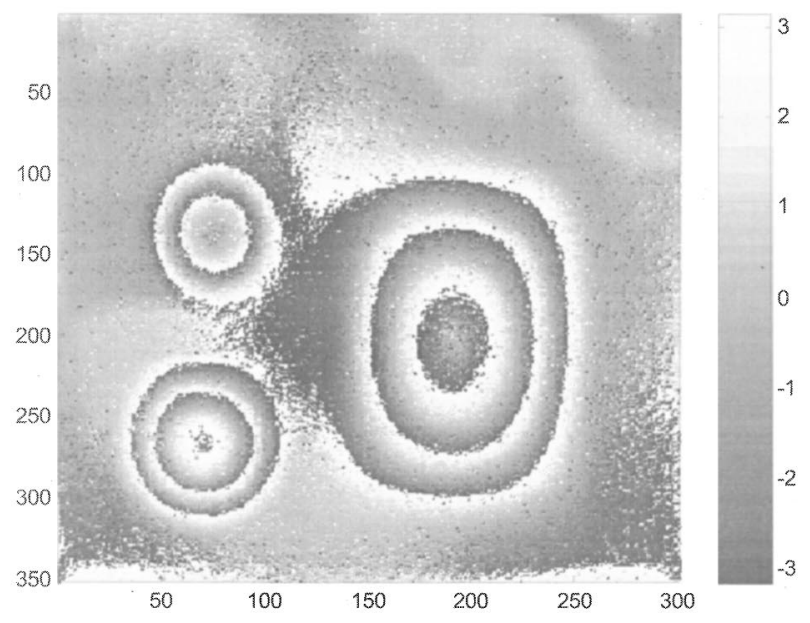

(c)

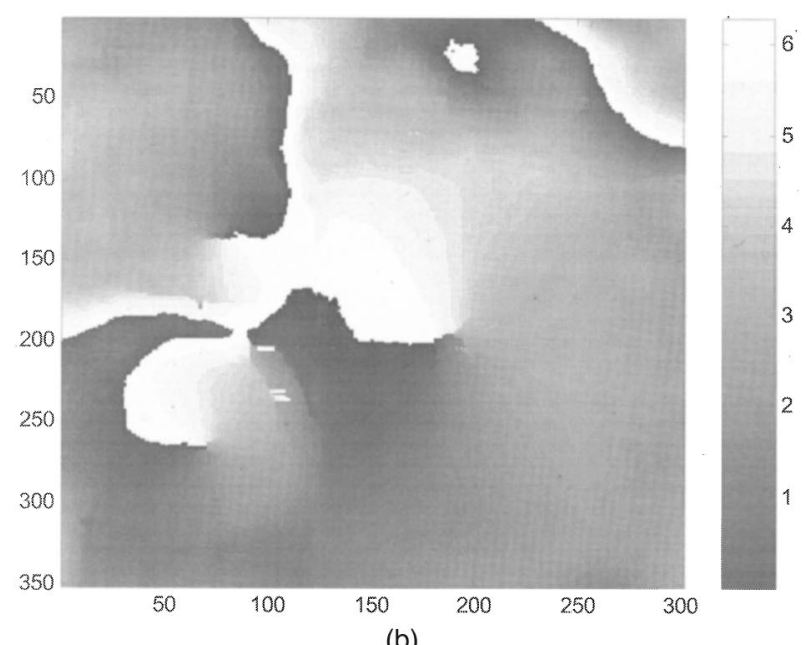

(b)

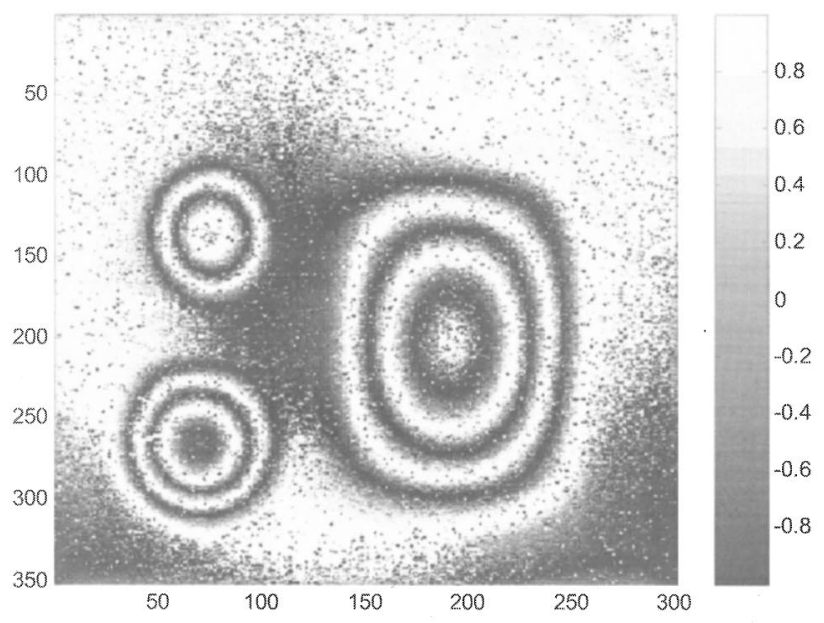

(d)

Fig. 9. (a) ESPI fringe pattern obtained in a deformation measurement experiment. (b) Modulo $2 \pi$ fringe orientation angle $\beta_{2 \pi}$ obtained by the modified RPT method for the fringe pattern of (a). (c) Demodulated phase map obtained by using the SPQ transform [relation (6)] applied to the fringe pattern of (a) with the orientation angle in (b). (d) Cosine of the phase map shown in (c); note the closeness with the fringe pattern shown in (a).

\section{ACKNOWLEDGMENTS}

We are grateful for the financial support of this work given by the European Union under project INDUCE, Contract BRPR-CT97-0805, and by the National Council for Science and Technology (CONACYT), Mexico, under project PROSUVE, contract PR48/01-9858.

The corresponding author, Juan Antonio Quiroga Mellado, may be reached by e-mail at aq@fis.ucm.es.

\section{REFERENCES}

1. T. Kreis, Holographic Interferometry (Akademie, Berlin, 1996).

2. N. Alcalá-Ochoa, J. L. Marroquin, and A. Dávila, "Phase recovery using a twin pulsed addition fringe pattern in ESPI," Opt. Commun. 163, 15-19 (1999).

3. J. A. Quiroga, J. A. Gomez-Pedrero, and A. Garcia-Botella, "Algorithm for fringe pattern normalization," Opt. Commun. 197, 43-51 (2001).

4. X. Zhou, J. P. Baird, and J. F. Arnold, "Fringe-orientation estimation by use of a Gaussian gradient-filter and neighboring-direction averaging," Appl. Opt. 38, 795-804 (1999).

5. M. Servin, J. L. Marroquin, and F. J. Cuevas, "Fringefollower regularized phase tracker for demodulation of closed-fringe interferograms," J. Opt. Soc. Am. A 18, 689695 (2001).

6. J. L. Marroquin, R. Rodriguez-Vera, and M. Servin, "Local phase from local orientation by solution of a sequence of linear systems," J. Opt. Soc. Am. A 15, 1536-1544 (1998).

7. K. G. Larkin, D. J. Bone, and M. A. Oldfield, "Natural demodulation of two-dimensional fringe patterns. I. General background of the spiral phase quadrature transform," J. Opt. Soc. Am. A 18, 1862-1870 (2001).

8. R. N. Bracewell, The Fourier Transform and Its Applications, 2nd ed. (McGraw Hill, New York, 1978).

9. D. Ghiglia and M. D. Pritt, Two-Dimensional Phase Unwrapping (Wiley, New York, 1998).

10. M. Servin, F. J. Cuevas, D. Malacara, J. L. Marroquin, and R. Rodriguez-Vera, "Phase unwrapping through demodulation by use of the regularized phase-tracking technique," Appl. Opt. 38, 1934-1941 (1999).

11. B. Ströbel, "Processing of interferometric phase maps as complex-valued phasor images," Appl. Opt. 35, 2192-2198 (1996). 